\title{
Braquiterapia de baja tasa en el tratamiento del cáncer de próstata localizado
}

\author{
Budía Alba A, Bosquet Sanz M, Tormo Micó A*, Boronat Tormo F, Alapont Alacreu JM, \\ Francés A*, Vera Donoso CD, Jiménez Cruz JF.
}

Servicios de Urología y Oncología Radioterápica*. Hospital Universitario La Fe de Valencia.

Actas Urol Esp. 2007;31(5):452-468

\section{RESUMEN}

BRAQUITERAPIA DE BAJA TASA EN EL TRATAMIENTO DEL CÁNCER DE PROSTATA LOCALIZADO

Introducción y Objetivo: La braquiterapia prostática se ha consolidado en los últimos años como tratamiento de primera línea en pacientes seleccionados con cáncer de próstata localizado. Pretendemos realizar una actualización del tema.

Material y Métodos: Revisión bibliográfica de las principales series publicadas analizando, indicaciones (selección de pacientes), las diferentes técnicas de implantación, los resultados oncológicos en función de grupos de riesgo y las complicaciones agudas y crónicas.

Resultados: La supervivencia libre de recaída bioquímica fue del 87-96\% a 10 años en tumores de bajo riesgo y del 63-86\% a 10 años en tumores de riesgo intermedio. Las principales complicaciones fueron la retención urinaria en el 3-24\%, necesitando RTU de próstata en 0-8,7\%, la incontinencia urinaria en el 0-6,7\%, proctitis en 0$15,5 \%$, disfunción eréctil en 6,3-30\%, úlceras/fístulas rectales en 0-5,4\%.

Conclusiones: La braquiterapia prostática constituye una alternativa terapéutica en el cáncer de próstata localizado de bajo riesgo y en pacientes seleccionados de riesgo intermedio, con escasas complicaciones y una aceptable tasa de control bioquímico.

Palabras clave: Braquiterapia prostática. Cáncer de próstata.

\begin{abstract}
INDICATIONS, RESULTS AND TECHNIQUES OF PERMANENT PROSTATE BRACHITHERAPY FOR LOCALIZED PROSTATE CANCER

Introduction and Objective: Prostate brachytherapy is a first-line therapeutic approach for localized prostate cancer in selected patients. We present our experience in brachytherapy and a thorough review of the literature.

Materials and Methods: A review of the literature and evaluation of patient's selection was done. Furthermore the implantation technique, oncological results according to the different risk groups and acute and chronic complications were also analyzed.

Results: The biochemical relapse-free 10 year survival rate was $87-96 \%$ in low risk tumours and $63-86 \%$ in intermediate risk tumours. A total of 3-24\% underwent urinary retention that required TURP in $0-8,7 \%$. Other complications were urinary incontinence in $0-6,7 \%$, proctitis in $0-15,5 \%$, erectile dysfunction in $6,3-30 \%$, rectal ulcer/fistula in 0-5,4\%.

Conclusions: Prostate brachytherapy is a safe and effective treatment in low and intermediate risk patients with prostate cancer.
\end{abstract}

Keywords: Prostate brachytherapy. Prostate cancer.

$\mathrm{L}^{2}$ a braquiterapia prostática implica la colocación de fuentes radiactivas en la glándula prostática. Dado su corto rango de irradiación emitido, altas dosis de energía pueden ser liberadas por estas fuentes dentro de la próstata, evitando una excesiva irradiación a órganos veci- nos. El desarrollo de nuevas tecnologías ha permitido mejorar progresivamente no sólo la colocación de las semillas radiactivas, sino también la distribución de la dosis de irradiación. Como resultado de todo ello, la braquiterapia prostática transperineal con implante permanente constituye 
una opción definitiva de tratamiento en pacientes con un cáncer de próstata clínicamente localizado. Las tasas de control bioquímico en pacientes tratados mediante esta técnica son similares a aquellos tratados mediante radioterapia externa o prostatectomía radical $^{1}$.

\section{PERSPECTIVA HISTÓRICA}

La braquiterapia representa una de las técnicas más antiguas de radiación para el cáncer de próstata. Las primeras referencias históricas datan del año 1911, en el que Pasteau ${ }^{2}$ describió el implante de radio intraprostático vía transuretral mediante un catéter. Posteriormente, con los malos resultados obtenidos, la alta tasa de complicaciones (20\%) y el desarrollo de otras alternativas para el tratamiento del cáncer de próstata como la cirugía, la manipulación hormonal y la radiación externa en 1956, esta técnica se abandonó. No es hasta 1972 cuando se describió una técnica retropúbica abierta con $\mathrm{I}^{125} 3 \mathrm{y}$ con oro $\mathrm{Au}^{198} 4$, pero los malos resultados clínicos junto con la distribución heterogénea de las dosis en la glándula con el implante manual la llevó a un segundo plano. Sin embargo hasta 1983 no se retoma la técnica, con un nuevo abordaje transperineal cerrado. En dicho año, Holm et al. ${ }^{5}$ reportan su método mediante un abordaje transperineal cerrado para la braquiterapia prostática (fuentes de $\mathrm{I}^{125}$ ) con un template antiguo y combinada con una imagen ecográfica obtenida vía transrrectal. Este abordaje fue posteriormente modificado por investigadores en Seattle, aplicando esta modalidad de tratamiento a sus primeros pacientes en Noviembre de 1985. Desde entonces, el refinamiento de la técnica así como las mejoras en el equipamiento han permitido que la braquiterapia se establezca como una alternativa fiable de tratamiento en el cáncer de próstata organoconfinado. En 1995 Stone y Stock $^{6}$ describen la técnica a tiempo real y desarrollan los nomogramas para cargas periféricas.

\section{SELECCIÓN DE PACIENTES}

La Sociedad Americana de Braquiterapia (ABS) recomendó la braquiterapia como monoterapia en pacientes con estadios clínicos $\mathrm{T} 1-\mathrm{T} 2 \mathrm{a}$, PSA menor o igual a $10 \mathrm{ng} / \mathrm{mL}$ y Gleason igual o menor de 6. La radioterapia externa debía ser asociada al implante en todos aquellos pacientes con factores de alto riesgo, es decir que no cumplieran los criterios descritos ${ }^{7}$. Sin embargo, la experiencia y un incremento en el número de datos científicos han permitido evitar en muchas ocasiones la radioterapia externa en función de la calidad del implante y la ubicación de las semillas. Así, de acuerdo a las recomendaciones de la ESTRO/EAU/EORTC/ABS el tratamiento con braquiterapia está indicado en pacientes con adenocarcinoma de próstata con PSA $\leq 10$ $\mathrm{ng} / \mathrm{mL}$, Gleason $\leq 6$ y estadio clínico T1c-T2a. Será opcional en tumores con riesgo intermedio en los que se cumpla al menos 1 de las siguientes condiciones; PSA 10-20 ng/mL o Gleason 7 o estadio clínico T2b-T2c. Para tumores de alto riesgo, PSA > $20 \mathrm{ng} / \mathrm{mL}$, Gleason 8-10 y/o estadio clínico T3, el tratamiento con braquiterapia es investigacional o debe aplicarse combinado con RT externa. Está contraindicado en pacientes con esperanza de vida inferior a 5 años o con enfermedad metástasica.

Analizamos a continuación distintas variables a considerar a la hora de seleccionar pacientes candidatos a tratamiento con braquiterapia:

- Tamaño prostático: El tamaño prostático en sí mismo no es una contraindicación relativa para el implante, como se creía en un principio, al pensar que era técnicamente más difícil y que asociaba una mayor morbilidad urinaria. Aportaciones recientes indican que próstatas grandes pueden ser implantadas con una aceptable morbilidad ${ }^{8,9}$. Así, Merrick et al. no encontraron asociación entre el volumen prostático y el análisis dosimétrico de la calidad del implante ${ }^{10}$. En próstatas grandes, la interferencia del arco púbico en la inserción de las agujas puede solucionarse con maniobras sencillas, colocando al paciente en una posición de litotomía extendida o dirigiendo las agujas alrededor del arco púbico.

- Edad: La tolerabilidad de la braquiterapia es similar para pacientes jóvenes y añosos. Su aplicación en pacientes jóvenes ha demostrado excelentes tasas de supervivencia libres de recidiva biológica a 7 años de seguimiento ${ }^{11}$. Solo está contraindicada la braquiterapia cuando la esperanza de vida es inferior a 5 años.

- IPSS pre-implante: Aunque los pacientes con mayor clínica obstructiva miccional preimplante 
son los que mayor riesgo de retención urinaria postimplante tienen, la relación entre el IPSS preimplante y la obstrucción urinaria post-implante es algo controvertida. Así, Terk et al. ${ }^{12}$ relacionaron el IPSS categorizado con el riesgo de retención urinaria, así a mayor IPSS, mayor riesgo de retención. Sin embargo, Merrick et al. utilizando tratamiento con $\alpha$-bloqueantes previo y mantenido tras el implante no encontraron correlación entre el IPSS y el riesgo de retención urinaria ${ }^{13}$. Un estudio prospectivo encontró una correlación débil entre el IPSS y el riesgo de retención urinaria o disfunción miccional. En este estudio ni los datos urodinámicos, ni el residuo postmiccional, ni el flujo máximo, ni la uretrocistoscopia preimplante predijeron la retención urinaria ${ }^{14}$.

- Prostatitis: No existe evidencia que la presencia histológica de prostatitis tenga relación con la morbilidad urinaria posterior al implante ${ }^{15}$.

- RTU-P previa: Desde la aplicación del tipo de implante periférico, limitando la dosis aplicada a la uretra, el riesgo de incontinencia en estos enfermos ha disminuido notablemente. La tasa de incontinencia en estos pacientes es inferior al $6 \%{ }^{16}$. La resección transuretral previa no contraindica la braquiterapia siempre y cuando exista suficiente tejido prostático para implantar semillas.

-Variables histológicas de mal pronóstico: Tumores con alto grado de Gleason, invasión perineural y alta carga tumoral en la biopsia se han correlacionado con alta probabilidad de extensión extracapsular. Sin embargo, basándonos en resultados obtenidos del estudio de las piezas de prostatectomía radical, se mostró que en aquellos pacientes en los que existía invasión capsular, en la mayoría esta extensión no superaba los $5 \mathrm{~mm}^{17}$. Este dato anatomo-patológico indica que en estos casos la braquiterapia sola o combinada con radioterapia externa podría esterilizar la extensión extraprostática, dado que un margen de $4 \mathrm{~mm}$ extracapsular es cubierto por el implante de semillas radiactivas. Así, distintas series publicadas han obtenido buenos resultados en estos pacientes de alto riesgo mediante una técnica agresiva locorregional. Estas técnicas combinan la braquiterapia con radiación externa, planificando el volumen diana con amplios márgenes periprostáticos con liberación de dosis terapéuticas de irradiación a la glándula prostática, región extracapsular y vesículas seminales, obteniendo supervivencias actuariales libres de recaída biológica a 5 años superiores al $70 \%{ }^{18-20}$. Buenos resultados se han obtenido también en pacientes con tumores con un Gleason 8-10, mediante tratamiento con radioterapia externa, reduciendo su probabilidad de falecimento ${ }^{21}$. Do et al., también encontraron en tumores de alto riesgo que la prostatectomía radical combinada con irradiación externa, mostraba mayor probabilidad de supervivencia que ambas técnicas por separado (probabilidad de supervivencia libre de enfermedad a 5 años del $80 \%$ para cirugía mas radioterapia, 60\% para radioterapia y $35 \%$ para cirugía $)^{22}$. Así, formas histológicas agresivas no parecen ser una contraindicación absoluta para la braquiterapia, pero en estos casos se debe considerar la combinación con irradiación externa. Si la opción de tratamiento elegida es la braquiterapia en monoterapia, ésta requerirá una técnica meticulosa con unos márgenes periprostáticos amplios con la colocación de semillas en localización extraprostática. La dosimetría postimplante deberá confirmar la correcta irradiación de la glándula prostática y de la regiones extracapsulares ${ }^{23}$.

-Obesidad: La obesidad no suele suponer, a diferencia de la cirugía abierta o la RT externa, mayores dificultades técnicas para su realización ni modifica los resultados a largo plazo ${ }^{24}$.

- Enfermedad inflamatoria intestinal: La colitis ulcerosa y la enteritis son contraindicaciones relativas para el tratamiento con radioterapia. Sin embargo, Grann et al. no evidenciaron morbilidad gastrointestinal asociada al implante con braquiterapia con $\mathrm{I}^{125}$ en pacientes con estas afecciones $^{25}$.

\section{TÉCNICA DE IMPLANTACIÓN}

La implantación de semillas requiere anestesia regional o general. El implante puede ser temporal o permanente. En los implantes temporales las fuentes radioactivas son depositadas por un tiempo limitado en la glándula prostática y luego retiradas, a diferencia de los permanentes que son implantados de forma indefinida y cuya actividad decae de forma progresiva hasta un estado inerte en un tiempo predecible. 
Los implantes temporales eran realizados de forma exclusiva con Iridio 192. La mayoría de este tipo de implantes han sido de baja tasa y durante un máximo de tres días. También podían ser de alta tasa aplicados durante unas horas. Tradicionalmente los implantes temporales precisaban de una laparotomía y guías intraoperatorias de las agujas transperineales, pero actualmente al igual que ocurre en los implantes permanentes de baja tasa con semillas, las mejoras tecnológicas han permitido el uso de guías ultrasónicas evitando la laparotomía al paciente ${ }^{26}$.

Los implantes permanentes mediante el abordaje transperineal mejoró los resultados de las técnicas previas retropúbicas, al conseguir incrementar la uniformidad en la distribución de la carga o dosis, aportando mayor calidad al implante y reduciendo la morbilidad del tratamiento. Los avances tecnológicos más importantes han sido las mejoras en la imagen por ecografia transrrectal, que permitieron la visualización intraoperatoria de la inserción de las agujas dentro de la próstata. La posibilidad de visualización en tiempo real de la inserción de las agujas ha permitido aumentar la exactitud del implante y reducir la morbilidad de la técnica. También ha sido reducida al utilizar agujas más atraumáticas, finas e incrementar la exactitud de su colocación al utilizar un template transperineal rígido, con un diseño estandarizado en forma de grid con agujeros distribuidos de forma simétrica y paralelos entre ellos.

Los isótopos más frecuentemente utilizados en los implantes permanentes son el $\mathrm{I}^{125} \mathrm{y}$ el $\mathrm{Pd}^{103}$. El $\mathrm{Pd}^{103}$ es habitualmente usado para pacientes con alto riesgo en que esta técnica se combina con la irradiación externa o en pacientes con Gleason superior a $7^{27}$.

La elección de un isótopo u otro está en función de su energía y su vida media. La radiación de fuentes de alta energía como el Iridio 192 y el $\mathrm{Au}^{198}$ penetra más en el tejido, de tal forma que la posición de las fuentes es menos crítica para alcanzar una dosis homogénea. Sin embargo la mayor penetración dentro del tejido puede ir asociado a un mayor daño del tejido no patológico, causando potencialmente mayores complicaciones y limitando la dosis a aplicar al volumen diana. La irradiación con fuentes de baja o mode- rada energía tales como el $\mathrm{I}^{125}$ y el $\mathrm{Pd}^{103}$ (las más utilizadas) liberan una dosis más confinada a la próstata pero su colocación exige una mayor precisión para evitar áreas infradosificadas (áreas frías) debido a su menor penetración en el tejido. En implantes permanentes el más usado es el $\mathrm{I}^{125}$, caracterizándose por una baja energía $(27 \mathrm{keV})$ y una vida media de 60 días. Su actividad a los 6 meses es del 10\% y al año del $0 \%$. La ventaja de trabajar con una baja energía radica en la mayor radioprotección del personal médico y en una mayor protección del tejido sano que rodea la zona tratada. Sin embargo por este mismo motivo la ubicación de las fuentes debe ser lo más precisa posible, dado que el riesgo de una distribución no homogénea de las cargas es mayor. El $\mathrm{Pd}^{103}$ tiene una energía similar al $\mathrm{I}^{125}(21 \mathrm{keV})$ pero con una vida media más corta (17 días), lo que obliga a utilizar una tasa más alta y mayor número de semillas. Es útil en tumores pobremente indiferenciados con tasas de proliferación celular altas ${ }^{26}$. Su actividad a los 2 meses es del 10\% y a los 6 meses del $0 \%$.

Analizaremos a continuación el implante permanente como método de tratamiento del cáncer prostático organoconfinado.

\section{EFECTOS DE LA IRRADIACIÓN}

La fuente radiactiva emite fotones incidentes a nivel celular, que producen dos tipos de efectos:

a) Efecto directo: Los fotones son absorbidos por macromoléculas celulares (DNA, RNA, proteínas, enzimas) induciéndoles un daño irreversible y conduciendo a la célula a la muerte celular.

b) Efecto indirecto: La irradiación es absorbida por el medio en el que están suspendidas las moléculas (agua), disociando la molécula de agua en radicales tóxicos para la célula, llevándola a su muerte.

\section{IMPLANTE PERMANENTE}

Las partes de un implante permanente son:

1) Planificación: Se planifica la teórica distribución de las semillas.

2) Implante: La colocación de las semillas.

3) Postplanificación: Evaluación de la calidad del implante. 


\section{POSICIÓN DEL PACIENTE}

La realización de esta técnica precisa de anestesia general o raquídea. La posición del paciente es de litotomía dorsal forzada con previa cateterización vesical. El intensificador de imagen fluoroscópica con un brazo en $\mathrm{C}$ debe ser colocado sobre la vejiga y próstata. La imagen ecográfica es obtenida mediante una sonda biplanar transrrectal (Fig. 1).

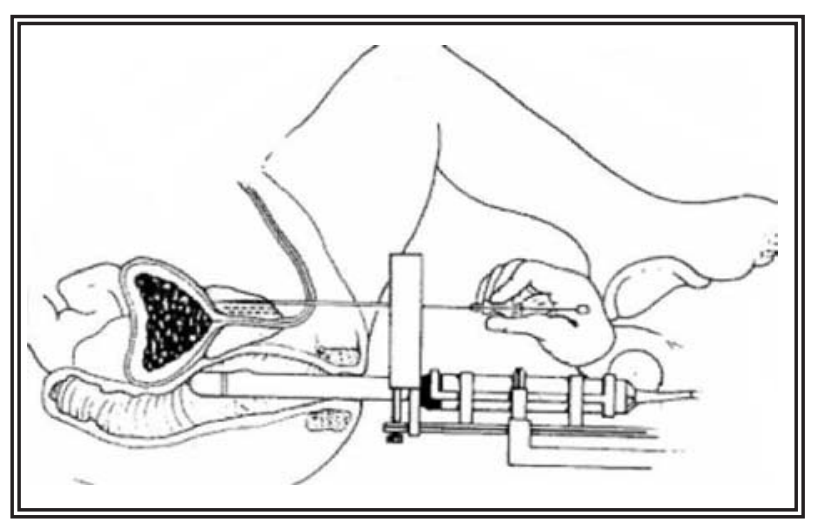

FIGURA 1. Sonda biplanar transrectal durante el implante permanente de semillas.

\section{FASES DEL IMPLANTE}

Planificación: La planificación se realiza mediante la obtención de la imagen ecográfica transrrectal de la próstata, determinando su volumen tras dividir en cortes ecográficos de 0,5 $\mathrm{cm}$ la próstata desde el ápex hasta la base. Esta información puede ser obtenida previa al procedimiento definitivo o intraoperatoriamente. Tras la obtención de la imagen, la distribución apropiada de las fuentes se determina por optimización de la dosis total de tratamiento obtenida con la ayuda de software específico, que delimita la dosis liberada en la próstata evitando que recto y uretra reciban excesiva irradiación. Las fuentes habitualmente se planifican a $1 \mathrm{~cm}$ unas de otras, debido a la limitación de los templates perineales que restringen el espacio de sus orificios a $0,5-1 \mathrm{~cm}^{1}$.

Implante. (procedimiento de braquiterapia): La imagen ecográfica transrrectal ayudada o no de una imagen fluoroscópica permite la visualización directa del proceso de inserción de la aguja. Las mejoras técnicas en la imagen ecográfica transrrectal permitiendo planos axiales y longitu- dinales, ayudan a la colocación exacta de las agujas. Una vez la aguja ha sido correctamente insertada (con las semillas a lo largo de su trayecto), las semillas son colocadas desde la base de la próstata hasta el ápex en la coordenada seleccionada. La próstata es un órgano elástico, móvil, por lo que una monitorización en el momento de la inserción de la aguja es necesaria ya que la propia inserción de la aguja produce un movimiento de distorsión que puede alterar la posición final de la aguja. La monitorización de la inserción permite reconocer y recolocar la aguja si fuera necesario.

Evaluación postimplante: La calidad del implante se evalúa mediante un análisis dosimétrico basado en imágenes obtenidas por TAC o RMN a las 4 semanas postimplante. Esta evaluación permite obtener información acerca de la cobertura y uniformidad del implante. También permite la posibilidad de comparar distintas técnicas intraoperatorias y proporciona una base teórica para mejorar la técnica de implantación. Aunque la TAC se realiza en la planificación prostática previa a irradiación externa, su uso en braquiterapia es controvertido. Dicha controversia surge de los cambios que aparecen tras el implante en el que el contorneo exacto de la próstata no es posible debido al edema y a la degradación de la imagen por el implante de semillas metálicas. Por otra parte existe una tendencia a sobrestimar el volumen prostático por parte del TAC respecto a la ecografía transrrectal. El momento de realizarlo también es controvertido. Algunos autores recomiendan su realización al $30^{\circ}$ día posterior al implante para permitir que el edema se haya reducido. Otros autores proponen su realización en el día 0 , cuando el edema postimplante es máximo, lo que permite un mayor aprendizaje de cara a mejorar la técnica de implantación. En técnicas intraoperatorias permite evaluar el implante de forma inmediata ${ }^{28,29}$. La mayoría de los autores miden la calidad del implante en términos de D90 (dosis recibida por el 90\% del volumen diana), V100 (porcentaje del volumen prostático que recibe el 100\% de la dosis) y V150 (porcentaje del volumen prostático que recibe el $150 \%$ de la dosis planificada), aunque la definición de calidad del implante permanece controvertida. Otros autores piensan que la 
dosimetría uretral y rectal debería ser incluida en la definición como variables predictoras de complicaciones $^{23}$. En nuestra unidad utilizamos en la evaluación dosimétrica postimplante al $30^{\circ}$ día, la fusión entre la imagen del TAC y la RMN, junto a la realización de una radiografía simple. La TAC localiza muy bien las semillas, pero no los límites prostáticos. La RMN gradiente localiza bien las semillas y la RMN $T_{2}$ proporciona una mejor visualización anatómica. La fusión se realiza haciendo coincidir las semillas de ambas técnicas, prescindiendo de las referencias óseas para la fusión. El cálculo dosimétrico lo realizamos con el software Simuplan.

\section{TIPOS DE TÉCNICAS}

Los tipos de técnicas de implantación permanente de semillas radiactivas vía transperineal son:

Técnica de la pre-planificación o "método de Seattle": El implante de las semillas va precedido de una preplanificación ambulatoria dos-tres semanas antes del implante. La preplanificación consiste en una ecografía transrrectal realizando cortes cada $5 \mathrm{~mm}$ desde la base hasta el ápex, con cálculo del volumen prostático por el software del ecógrafo. Las imágenes son transferidas a un ordenador para planificar la posición de las semillas y el cálculo de las dosis. El principal problema de esta técnica es la dificultad en reproducir la planificación, la necesidad de compensar el movimiento de la próstata durante el implante y la obligación de pinchar en coordenada prevista del preimplante. El implante de las semillas se realiza mediante un template, vía transperineal y tras haber reproducido el preplan, confirmando que la colocación de las mismas se realiza de acuerdo al plan previo. Las semillas son insertadas mediante agujas precargadas con las semillas unidas en hebras de ácido poliglicólico o mediante semillas sueltas con el aplicador de Mick. Este tipo de técnica no previene la posible interferencia del arco púbico y existe un mayor riesgo de complicaciones ante situaciones especiales como pacientes adenomectomizados o en rescate. La postplanificación se realiza 4 semanas después mediante TAC sin poder corregir las zonas no dosificadas adecuadamente a diferencia de la técnica a tiempo real ${ }^{30}$. Su principal des- ventaja es la discordancia que puede surgir entre la imagen obtenida en la planificación y el implante definitivo.

Planificación intraoperatoria interactiva a tiempo real: Es la técnica más novedosa en braquiterapia prostática y su principal ventaja es que permite realizar correcciones intraoperatorias, aumentando la exactitud del implante en un solo acto al colocar la aguja directamente sin obligación de seguir una coordenada (a diferencia del método anterior). Previo a la implantación el volumen prostático es medido mediante ecografia transrrectal con planos de $5 \mathrm{~mm}$ desde la base al ápex. La cantidad apropiada de actividad es calculada en base a tablas de volumen-actividad, planificando el implante en el propio quirófano. La actividad total obtenida de acuerdo al volumen es dividida por la actividad por semilla $(0,3-$ $0,5 \mathrm{mCi}$ por semilla para el $\mathrm{I}^{125}$ y 1,0 a $1,5 \mathrm{mCi}$ por semilla para el $\mathrm{Pd}^{103}$ ), obteniendo el número total de semillas a implantar. Tras determinar el número de semillas a implantar se realizan cortes ecográficos transversales y longitudinales a la próstata, midiendo las distancias anteriores y posteriores a la uretra. Estas medidas permiten determinar el espacio entre las semillas en el implante. Este tipo de implante permite realizar una postplanificación intraoperatoria por ecografia sin necesidad de realizar una TAC a las 4 semanas, aunque otros autores lo recomiendan. Por todo ello el número de complicaciones es menor y el manejo de situaciones especiales como pacientes adenomectomizados también se asocia a un menor número de complicaciones ${ }^{30}$. $\mathrm{Su}$ principal desventaja es el tiempo quirúrgico que consume y la imposibilidad de tener preparadas las semillas exactas a implantar para un determinado paciente.

\section{TIPO DE IMPLANTE}

Los implantes pueden ser uniformes a lo largo de toda la glándula, periféricos respetando la zona central periuretral o con criterios de ambos (periférico modificado). En la actualidad el implante uniforme de las fuentes ha sido abandonado en favor del periférico o el periférico modificado. El más utilizado es el periférico modificado en el que la mayor carga recae en la zona periférica, reduciendo la carga en la zona periu- 
retral, a diferencia del periférico en el que todas las cargas recaen estrictamente en la zona periférica. Sin embargo en las técnicas actuales el cálculo conformacional de la dosimetría del implante se realiza de forma individualizada y adaptada a la anatomía de la próstata a tratar, en lugar de un cálculo arbitrario sobre un volumen diana ${ }^{1}$.

\section{RESULTADOS DE LA BRAgUITERAPIA DE BAJA TASA}

La mayoría de las publicaciones de braquiterapia prostática muestran resultados oncológicos comparables a los más positivos de series de prostatectomía radical y radioterapia externa, con bajas tasas de incontinencia, lesión rectal y con aceptables tasas de disfunción sexual ${ }^{31}$. A causa de la existencia hoy en día de múltiples opciones efectivas de tratamiento para el cáncer de próstata localizado y la ausencia de ensayos clínicos prospectivos, existe numerosas controversias de acuerdo a las ventajas de un tratamiento sobre otro. Del mismo modo, en las distintas series de braquiterapia publicadas, existen diferencias en los criterios de selección de los pacientes, en las definiciones de recaída biológica, en las variantes técnicas utilizadas así como diferencias en la experiencia de los facultativos que la realizan. Por ello, desde una perspectiva basada en la evidencia, el valor de los resultados o la comparación de los mismos deben ser considerados con limitaciones. Serían necesarios estudios multicéntricos, prospectivos y aleatorizados.

$\mathrm{El}$ análisis de los resultados lo analizaremos en función del riesgo del tumor. Los resultados de las principales series se muestran en las Tablas 1,2 y 3.

- Tumores de bajo riesgo: Se incluyen en este grupo tumores de Gleason $\leq 6, \mathrm{PSA}<10 \mathrm{ng} / \mathrm{mL}$ y

Tabla 1

Supervivencia libre de recaída bioquímica tras tratamiento con braquiterapia prostática como modalidad única de tratamiento

\begin{tabular}{|c|c|c|c|c|c|c|c|}
\hline & $n^{\circ}$ pac & $\mathbf{P} / \mathbf{I}$ & T1/T2 (\%) & GS>6 (\%) & $\begin{array}{c}\text { PSA } \\
\text { (mediana) }\end{array}$ & $\begin{array}{c}\text { T. seguimiento } \\
\text { (mediana en meses) }\end{array}$ & $\begin{array}{l}\text { bSLE \% } \\
\text { (años) }\end{array}$ \\
\hline Blasko $(1995)^{37}$ & 197 & I & $19 / 81$ & 0 & 7.0 & 36 & $93(5)$ \\
\hline Beyer $(1997)^{79}$ & 489 & I & $13 / 87$ & 12 & 7,3 & 34 & $67-96(5)$ \\
\hline Stokes (1997) ${ }^{80}$ & 142 & I & $15 / 85$ & 0 & $10,6^{*}$ & 30 & $76(5)$ \\
\hline Grado $(1998)^{81}$ & 392 & $\mathrm{I} / \mathrm{P}$ & $6 / 92$ & 20 & 7,3 & 30 & $80(5)$ \\
\hline Sharkey (1998) 82 & 224 & $\mathrm{P}$ & $5 / 95$ & 26 & 7,4 & 27 & $50(3)$ \\
\hline Storey $(1999)^{83}$ & 193 & I & $24 / 76$ & 13 & 8,65 & $\mathrm{NE}$ & $63(5)$ \\
\hline Blasko (2000) ${ }^{18}$ & 230 & $\mathrm{P}$ & $30 / 70$ & 40 & 7,3 & 41,5 & $83,5(9)$ \\
\hline Radge $(2000)^{84}$ & 147 & I & $22 / 78$ & 0 & $8,8^{*}$ & 93 & $66(12)$ \\
\hline Zelefsky $(2000)^{33}$ & $248^{\wedge}$ & I & $58 / 42$ & 25 & 7 & 48 & $71(5)$ \\
\hline Brachman (2000) ${ }^{42}$ & 695 & $\mathrm{I} / \mathrm{P}$ & $17 / 83$ & 15 & $\mathrm{NE}$ & 51 & $64(7)$ \\
\hline Ragde $(2001)^{85}$ & 769 & $\mathrm{I} / \mathrm{P}$ & & & & 71 & $77(13)$ \\
\hline Grimm $(2001)^{32}$ & 125 & I & $24 / 76$ & 0 & 5,1 & 78 & $85(10)$ \\
\hline Kwok $(2002)^{39}$ & 102 & I & $29 / 71$ & 31,6 & ND & 74 & $57(5)$ \\
\hline Batterman (2004) ${ }^{40}$ & 351 & I & $58 / 42$ & $\mathrm{NE}$ & 10,8 & 48 & $64(5)$ \\
\hline Merrick (2004) ${ }^{11}$ & $119^{-}$ & $\mathrm{I} / \mathrm{P}$ & $\mathrm{NE}$ & $\mathrm{NE}$ & 6,9 & 65 & $98,3(7)$ \\
\hline Potters $(2005)^{34}$ & 1449 & $\mathrm{I} / \mathrm{P}$ & $61 / 39$ & 28 & 7,2 & 82 & $81(12)$ \\
\hline Merrick (2005) ${ }^{86}$ & 202 & $\mathrm{I} / \mathrm{P}$ & $\mathrm{NE}$ & $\mathrm{NE}$ & $\mathrm{NE}$ & 65 & $95,3(8)$ \\
\hline Stone $(2005)^{43}$ & $279^{\wedge \wedge}$ & I & $40 / 60$ & 2,2 & $\mathrm{NE}$ & 72 & $78(10)$ \\
\hline
\end{tabular}

bSLE: supervivencia libre de recaída bioquímica

I/P: Yodo 125, Paladio 103

* media PSA

$\wedge \wedge 64$ pacientes recibieron deprivación androgénica durante 5 ó 6 meses tras el implante.

^ 31 pacientes tratados con deprivación androgénica previo al implante.

- Sólo incluye pacientes de edad igual o menor de 62 años.

NE no especificad. 
Tabla 2

Supervivencia libre de recaída bioquímica tras tratamiento con braquiterapia prostática según grupos de riesgo

\begin{tabular}{|c|c|c|c|c|c|c|}
\hline & \multicolumn{2}{|c|}{ Bajo riesgo } & \multicolumn{2}{|c|}{ Riesgo intermedio } & \multicolumn{2}{|c|}{ Alto riesgo } \\
\hline & no pac. & bSLE(\%) & no pac. & bSLE(\%) & no pac. & bSLE(\%) \\
\hline D`Amico $(1998)^{38}$ & 32 & $88(5)$ & 15 & $32(5)$ & 19 & $\mathrm{NE}$ \\
\hline Stone $(1999)^{30}$ & 109 & $91(4)$ & 152 & $85-58(4)^{\wedge}$ & 40 & $71(3)^{\wedge}$ \\
\hline Blasco $(2000)^{18}$ & 103 & $92(5)$ & 107 & $82(5)$ & 20 & $65(5)$ \\
\hline Zelefsky $(2000)^{33}$ & 112 & $88(5)$ & 92 & $77(5)$ & 22 & $38(5)$ \\
\hline Grimm $(2001)^{32}$ & 97 & $87(10)^{*}$ & 27 & $79(10)^{*}$ & $\mathrm{NE}$ & $\mathrm{NE}$ \\
\hline Kwok $(2002)^{39}$ & 41 & $85(5)^{*}$ & 33 & $63(5)^{*}$ & 28 & $24(5)^{*}$ \\
\hline Merrick (2004) ${ }^{11-}$ & 59 & $98,3(7)$ & 39 & $100(7)$ & 21 & $95,2(7)^{\wedge}$ \\
\hline Batterman $(2004)^{40}$ & 116 & $91(5)$ & 114 & $75(5)$ & 121 & $54(5)$ \\
\hline Stone $(2005)^{43}$ & 146 & $91,3(10)$ & - & $\longrightarrow$ & 133 & $66,1(10)^{-}$ \\
\hline Potters $(2005)^{34}$ & 481 & $91(12)$ & 554 & $80(12)$ & 418 & $66(12)^{\wedge}$ \\
\hline
\end{tabular}

- Sólo incluye pacientes de edad igual o menor de 62 años.

* supervivencia estimada

“ 66 pacientes recibieron deprivación androgénica por volumen prostático alto o por ser de alto riesgo.

$\wedge$ RT adyuvante. En el grupo de riesgo intermedio de Stone (1999) la mitad recibieron RT externa adyuvante. En este mismo grupo, el 85 $\%$ corresponde a los que recibieron hormonoterapia adyuvante y el $58 \%$ a los que no la recibieron. En la serie de Potters et al. el $20 \%$ del grupo de alto riesgo recibieron RT.

Tabla 3

Principales series españolas

\begin{tabular}{lccccccc}
\hline & $\mathbf{n}^{\mathbf{0}}$ pac & P/I & T1/T2 (\%) & GS>6 (\%) & $\begin{array}{c}\text { PSA } \\
\text { (mediana) }\end{array}$ & $\begin{array}{c}\text { T. seguimiento } \\
\text { (mediana en meses) }\end{array}$ & $\begin{array}{c}\text { bSLE \% } \\
\text { (años) }\end{array}$ \\
\hline Prada $(2005)^{58}$ & 275 & I & NE & 6 & $9,65^{*}$ & $31^{*}$ & $99(5)$ \\
Aguiló $(2005)^{87}$ & 130 & I & $76 / 24$ & 2 & $6,38^{*}$ & $66^{*}$ & $88,7(2)$ \\
Guinot $(2005)^{* 88}$ & 130 & I & $61 / 39$ & 3 & 7,1 & 16 & $95(3)$ \\
Tormo $(2006)^{\wedge}$ & 96 & I & $59 / 41$ & 16,6 & 7 & $98,7(1)$ \\
\hline
\end{tabular}

* Media

* Datos no publicados (Fundación IVO de Valencia).

$\wedge$ Datos no publicados (HU La Fe de Valencia).

estadio clínico T1c-T2a de la clasificación de la AJCC de 1997. La braquiterapia aplicada a este grupo de tumores muestra una supervivencia libre de recidiva bioquímica entorno al $87-96 \%$ a 5-10 años de seguimiento según las series publicadas $^{11,18,32-37}$.

- Tumores de riesgo intermedio: Se incluyen en este grupo tumores de Gleason 7 o PSA entre 10 y $20 \mathrm{ng} / \mathrm{mL}$ o estadio clínico T2b-c (AJCC 1997). Blasko et al. reportaron para este grupo de riesgo una supervivencia libre de progresión del $82 \%$ a 9 años con $\mathrm{Pd}^{103}$. La combinación con radioterapia externa no incrementó a 5 años la supervivencia libre de recaída biológica ${ }^{18}$. Merrick et al. obtuvieron una supervivencia libre de enferme- dad a 6 años del 97\% mediante la combinación de la braquiterapia con radioterapia externa en pacientes menores de 62 años. En este grupo de pacientes no hubo diferencias en la supervivencia en el Gleason 7, con predominio o no del factor 4 $\left(90 \%\right.$ a 5 años de seguimiento) ${ }^{20}$. Otras series con braquiterapia como modalidad única de tratamiento en este grupo de riesgo, obtuvieron supervivencias libres de enfermedad del $32-82 \%$ a 5 años de seguimiento ${ }^{18,30,32,33,38-40}$.

- Tumores de alto riesgo: Se incluyen pacientes con tumores con 2 ó 3 factores de riesgo intermedio tumores con Gleason 8-10, PSA > 20 $\mathrm{ng} / \mathrm{mL}$ o estadio $>$ cT2c. En este grupo los resultados son dispares. Así, Dattoli et al. comunica- 
ron una supervivencia libre de enfermedad a 5 años del $76 \%$ en pacientes tratados con radioterapia externa y braquiterapia posterior con $\mathrm{Pd}^{103}$ 19. El mismo grupo observó una supervivencia libre de enfermedad a 5 años del $62 \%$ en pacientes con tumores con Gleason 8-10 y del 70\% para tumores con un PSA pretratamiento $>20$ $\mathrm{ng} / \mathrm{mL}^{41}$. Sin embargo otras series no obtuvieron resultados tan favorables para los grupos de alto e intermedio riesgo. Así, Brachman et al. obtuvieron una supervivencia libre de recaída biológica del $28 \%$ a 5 años para tumores con Gleason mayor de 7 y del 53\% para tumores con PSA pretratamiento entre $10-20 \mathrm{ng} / \mathrm{mL}$, utilizando la braquiterapia como tratamiento único ${ }^{42}$. En la misma línea D’Amico et al. observaron una supervivencia que no superaba el $32 \%$ a 5 años en pacientes con riesgo intermedio o alto ${ }^{38}$. En ninguno de estos dos trabajos se hace referencia a la dosimetría postimplante. Así pues, los resultados de la braquiterapia en monoterapia en este grupo de riesgo muestran supervivencias del 3865 a 5 años ${ }^{18,33,40}$. Con radioterapia adyuvante se han obtenido supervivencias del 66-95\% a más de 5 años según series ${ }^{30}$ y con hormonoterapia adyuvante del $66,1 \%$ a 5 años ${ }^{11,34,43}$. Hay que tener en cuenta que las aportaciones en este grupo de riesgo son más limitadas y con grupos de pacientes más heterogéneos.

\section{TRATAMIENTO HORMONAL ADYUVANTE}

Varios ensayos clínicos prospectivos han demostrado que el tratamiento hormonal adyuvante con dosis convencionales de radioterapia externa (65-70 Gy) en pacientes con tumores localmente avanzados de próstata mejoran las tasas de supervivencia libre de enfermedad y cáncer-especifica. Su beneficio probablemente sea debido a la imposibilidad de la radioterapia en monoterapia para terminar con volúmenes tumorales extensos ${ }^{44}$.

Sin embargo, en tratamientos con braquiterapia no existe evidencia clínica que la manipulación hormonal incremente las tasas de supervivencia libre de enfermedad y cáncer específica. Sólo estudios recientes y a muy corto plazo han mostrado un posible beneficio ${ }^{45}$. Su uso sólo se indica en monoterapia, para disminuir el volumen inicial de la próstata.

\section{RADIACIÓN EXTERNA COMBINADA CON BRAQUITERAPIA}

El motivo de asociar a la braquiterapia una irradiación externa radica en aumentar la cobertura tisular periprostática en tumores de alto riesgo. Inicialmente era utilizada en tumores con PSA pretratamiento $>10 \mathrm{ng} / \mathrm{mL}$, un estadio $\geq T_{2 b}$ y/o Gleason $\geq 7$. Sin embargo, la aplicación de radioterapia suplementaria ha sido cuestionada debido a los buenos resultados de la braquiterapia en monoterapia en pacientes con Gleason y/o PSA pretratamiento alto. Estos resultados se han conseguido mediante un implante con un generoso margen periprostático. En estos casos, la radioterapia suplementaria no parece aumentar la supervivencia libre de recaída bioquímica en tumores de bajo, intermedio y alto riesgo ${ }^{18,20,35,37,42}$ con curvas a 5 años libres de progresión bioquímica superponibles a las de braquiterapia como única modalidad de tratamiento ${ }^{18,36,37}$.

\section{PSA SPIKES}

Se define con este término a una elevación temporal del PSA que ocurre en un tercio de los pacientes, entre los 12 y 30 meses posteriores al implante. Su mecanismo de producción probablemente sea un daño de la membrana celular del epitelio glandular secundario a una inflamación inducida por la radioterapia. Estas elevaciones de PSA no muestran un impacto negativo en la recaída bioquímica a largo plazo ${ }^{41,46}$.

\section{COMPLICACIONES DE LA BRAQUITERAPIA}

Los efectos secundarios de la braquiterapia pueden ser clasificados en dos grandes grupos en función del tiempo de aparición: aquellos que aparecen durante el primer año después del implante (complicaciones agudas o a corto plazo), y los que lo hacen tras el primer año de seguimiento (complicaciones crónicas o a largo plazo) ${ }^{47}$.

\section{COMPLICACIONES A CORTO PLAZO}

Están causadas por los efectos agudos de la radiación y por el traumatismo que se produce durante el implante de las semillas. El implante de las semillas puede producir un hematoma perineal subcutáneo, equimosis, edema y sangrado 
perineal. Si durante la colocación de las agujas se punciona el cuello vesical o la uretra, puede aparecer hematuria e incluso retención urinaria aguda (RAO) por coágulos ${ }^{47}$. La morbilidad debida a la radiación aguda está en función de la dosis recibida por la uretra, la próstata, el recto y los haces neurovasculares. Ésta será mayor en intensidad y frecuencia en los pacientes que reciben braquiterapia combinada con radioterapia externa. Suele manifestarse en el primer-tercer mes tras el implante y luego va disminuyendo progresivamente a medida que la radioactividad del isotopo se reduce. Cada isótopo tiene unas características radiobiológicas distintas, lo que puede condicionar el momento de aparición o el pico de intensidad de los sintomas.

\section{a) Complicaciones urológicas agudas}

- Sindrome miccional obstructivo y retención urinaria: Suelen aparecer en el 50\% de los pacientes. Se manifiestan principalmente con disuria y debilidad del chorro miccional, lo que ocasiona un deterioro significativo en el flujo medio y máximo de la flujometría libre urinaria y un aumento en el IPSS. Los cambios observados en la medición del residuo post-miccional son menos significativos ${ }^{48}$.

Los cambios más significativos en el IPSS suelen aparecer en la fase aguda y progresivamente se reducen en 1 ó 2 años hasta alcanzar el nivel previo $^{49}$. Los principales factores de riesgo implicados en las disfunciones de vaciado previo al implante son, un IPSS alto (>8) y la necesidad de tratamiento con $\alpha$-bloqueantes previo a la braquiterapia ${ }^{48}$. Terk et al. ${ }^{12}$ demostraron que el riesgo de retención urinaria era del $2 \%$ si el IPSS pre-implante era $<10$, del $11 \%$ si el IPSS estaba entre $10-19$ y del $29 \%$ si el IPSS era $>20$. Gelblum $^{50}$ relacionó al volumen prostático ( $>35$ $\mathrm{cm} 3$ ) con el riesgo de retención urinaria, aunque para otros autores ni el volumen de la próstata, ni la edad, ni el estadio clínico, ni el PSA, ni el Gleason mostraron relación con el riesgo de retención urinaria ${ }^{12,48}$. La RAO ocurre habitualmente al día siguiente del implante y probablemente está relacionada con el edema postimplante y los efectos de los agentes anestésicos, ya que la radiación liberada es todavía insignificante $^{12}$. Pese a todo ello, el índice de retenciones urinarias es bajo, entorno al 1,5-27\% ${ }^{12,47,50-53}$, y se suele resolver con la colocación de una sonda vesical por espacio de unas semanas y tratamiento médico con $\alpha$-bloqueantes. Pocos de estos pacientes $(0-8,7 \%)$ requerirán una resección transuretral de próstata (RTU-P) desobstructiva por RAO refractaria.

En caso de tener que llevar a cabo una RTU-P hay que esperar un tiempo mínimo de seguridad después del implante, unos seis meses para el $\mathrm{I}^{125} \mathrm{y}$ dos meses para el $\mathrm{Pd}^{103}$, tiempo en el que el 90\% de la radiación ha sido emitida. La RTU-P a fin de evitar la incontinencia urinaria postimplante, debe ser cuidadosa, comenzando la resección por el lóbulo medio y posteriormente resecando los lóbulos laterales. Se debe intentar conservar el cuello vesical a las 5 y las $7 \mathrm{~h}$ para preservar la principal irrigación de la uretra prostática. La resección del tejido de los lóbulos laterales debe ser mínima, teniendo extremo cuidado con la resección del ápex, quitando una cantidad mínima de tejido sólo si es necesario, ya que podría estar ya comprometido por la exposición a la radiación previa ${ }^{47,54}$. Mediante la RTU-P se obtendrán una media por resección de 5 a 10 semillas del interior de la próstata. Cuando una semilla es expuesta en la resección o el movimiento de corte con el asa de resección nota resistencia, se debe realizar un corte más profundo recolocando el asa. Esta maniobra facilita la retirada posterior de las semillas con el evacuador Ellik. Las semillas deben ser recogidas y entregadas en la oficina de seguridad de radiación para una eliminación adecuada. Posteriormente debe dejarse una sonda de 20-22 Fr durante una media de 2 días $^{54}$. La RTU-P no debe ser inmediata a la retención para diferenciar los pacientes con una retención urinaria refractaria de los que presentan una retención prolongada, en los que el mantenimiento por un tiempo prolongado de la sonda vesical resolverá el cuadro ${ }^{12}$.

Las tasas de incontinencia post-RTU-P tras braquiterapia oscilan entre el 18-70\% según las series publicadas (Tabla 4). El tiempo óptimo de espera para una RTU-P tras tratamiento con radiación es controvertido. Así, estudios realizados en pacientes tratados con radioterapia externa sugieren que la tasa de incontinencia post RTU-P aumenta cuanto menor sea el tiempo 
Tabla 4

Incontinencia urinaria post RTU-P en pacientes tratados con braquiterapia prostática

\begin{tabular}{lcccc}
\hline & $\mathbf{n}^{\mathbf{0}}$ pac & Tratamiento & $\begin{array}{c}\text { Incontinencia } \\
\text { n (\%) }\end{array}$ & Correlación \\
\hline $\mathrm{Hu}^{89}$ & 10 & Implante I ${ }^{125}$ & $7(70)$ & Dosis máx uretral $>400 \mathrm{~Gy}$ \\
Gelblum $^{50}$ & 28 & Implante $\pm \mathrm{RT}$ & $5(17)$ & ninguna \\
Koutrouvelis $^{90}$ & 11 & Implante I ${ }^{125}$ o Pd & ninguna \\
Kollmeier $^{54}$ & 38 & Implante I ${ }^{125} \mathrm{o} \mathrm{Pd}^{103} \pm \mathrm{RT}$ & $7(27)$ & ninguna \\
\hline
\end{tabular}

RT: radioterapia externa

transcurrido desde la radiación ${ }^{55}$. Sin embargo, Kollmeier et al., observaron tasas de incontinencia mayores en pacientes tratados con braquiterapia y RTU-P posterior, cuando ésta se realizaba a partir de los dos años postimplante. Los pacientes con retención secundaria al edema agudo del implante tenían menor probabilidad de retención tras una RTU-P que aquellos que presentaban síntomas obstructivos tardíos (tras 2 años) probablemente secundarios a la fibrosis inducida por la irradiación ${ }^{54}$. Sin embargo la principal limitación de este estudio es su corta muestra (38 pacientes con RTU-P), por lo que son necesarios más estudios, con mayor número de pacientes, que permitan aclarar el tiempo óptimo de espera previo a una RTU-P.

$\mathrm{El}$ uso de un $\alpha$-bloqueante no reduce el riesgo de retención prolongada (más de 3 días) ni reduce la necesidad de una cirugía desobstructiva postimplante ${ }^{49}$. Sólo aquellos pacientes que recibieron el tratamiento con un $\alpha$-bloqueante de forma profiláctica, alcanzaron antes el nivel basal de IPSS previo al implante que aquellos que no lo recibieron o lo recibieron de forma terapéutica ${ }^{13}$.

- Sindrome miccional irritativo: Es, sin duda, el efecto secundario más frecuente, presente en el $46-66 \%$ de los pacientes ${ }^{48,56}$, y se manifiesta como escozor al orinar, frecuencia-urgencia, nicturia acompañado en ocasiones de hematuria.

La sintomatología miccional secundaria a la radiación recibida sobre vejiga es clasificada por la Radiation Therapy Oncology Group (RTOG) en cinco grados. La toxicidad grado 0 implica la ausencia de cambios y la toxicidad grado 1 indica una ligera atrofia epitelial o una telangiectasia menor, pudiendo haber microhematuria. En la toxicidad grado 2 existe una telangiectasia generalizada, frecuencia miccional moderada y hema- turia macroscópica intermitente. En la grado 3 la frecuencia miccional es severa, con escozor intenso al orinar, telangiectasia generalizada severa (a menudo con petequias), hematuria frecuente, y puede existir reducción de la capacidad vesical $(<150 \mathrm{ml})$. En la toxicidad grado 4 hay necrosis, cistitis hemorrágica severa y reducción de la capacidad vesical (capacidad $<100 \mathrm{ml}$ ) (57). Esta escala es una valoración subjetiva y no está universalmente aceptada, aunque probablemente sea la más utilizada en la literatura.

Prada et al. ${ }^{58}$ clasifica a los pacientes con esta clínica en tres grupos en función de su comportamiento: un tercio de sus pacientes presentan una clínica insignificante, otro tercio presenta un sindrome irritativo-obstructivo que se resuelve con hábitos higiénico-dietéticos, o bien con la toma de antiinflamatorios o $\alpha$-bloqueantes de forma esporádica, y el otro tercio restante presenta una clínica de mayor intensidad, grado 1-2 de la RTOG, que obliga a tratamiento antiinflamatorio y $\alpha$-bloqueante pautado.

La relación de la sintomatología urinaria con la dosis recibida por la próstata permanece controvertida. Así Desai et al. ${ }^{49}$ relacionaron la elevación del IPSS con la actividad total implantada (V80/90/95/100 y D70/90/100/150). Sin embargo, Merrick et al. ${ }^{8}$ no encontraron correlación entre el IPSS y la dosis uretral o las dosis totales prostáticas aplicadas. En esta misma línea, Wallner et al. ${ }^{59}$ relacionaron la toxicidad urinaria con una máxima dosis uretral mayor al $250 \%$ de la dosis mínima periférica. Actualmente con los implantes periféricos rara vez la dosis uretral supera el $150 \%$ de la dosis prescrita. Por ello la recomendación es que la dosis uretral esté entre el 100-140\% de la dosis prescrita. 
La clínica aparece entre el primer y tercer mes para luego disminuir de forma progresiva, de tal forma que alrededor del 66-92\% de los pacientes tendrán una mejoría de esta clínica con el tiempo hasta ser mínima o indetectable en el $6^{-}-12^{\circ}$ mes $(48,58)$. No obstante puede que algunos pacientes experimenten un cuadro de reagudización de la clínica una vez desaparecida la misma, habitualmente de corta duración y menor intensidad, o esta clínica no desaparezca y permanezca presente. Wallner et al. ${ }^{56}$, detectó a los dos años del implante que un $14 \%$ de sus pacientes tenían sintomatología urinaria persistente grado $\geq 2$ de la RTOG.

- Disconfort en la eyaculación, disminución del volumen del eyaculado y eyaculación retrógrada: aparece en un bajo de porcentaje de los pacientes y suele desaparecer por completo en todos los enfermos con el paso del tiempo.

\section{b) Complicaciones digestivas agudas}

La manifestación más frecuente es la proctitis, que cursa con un aumento del número de deposiciones en una o dos veces con respecto al hábito intestinal normal, cierto grado de tenesmo rectal, deposiciones con mucosidad, prurito y pesadez en la zona anal. Al igual que para la clínica miccional, la RTOG clasifica en cinco grados la toxicidad rectal. En la toxicidad grado 0 no existen cambios y en la toxicidad grado 1 se produce diarrea o rectorragia leve, con hasta cinco deposiciones al día. En la toxicidad grado 2 se produce una diarrea moderada, con más de cinco deposiciones al día, mucosidad rectal excesiva o rectorragia intermitente y en la grado 3 se produce obstrucción o rectorragia que precisa intervención quirúrgica para su resolución. En la grado 4 se produce necrosis o perforación de la mucosa con la aparición de fístulas ${ }^{57}$. El pico máximo de aparición de esta sintomatología es más tardío que el de la sintomatología urinaria, situándose en los ocho meses $^{58}$ y tiene una incidencia del $2 \% 56,58$, aunque en algunas series alcanza el $15 \%{ }^{51}$, suele ser auto-limitada.

\section{COMPLICACIONES A LARGO PLAZO}

a) Complicaciones urológicas a largo plazo

- Estenosis uretral o del cuello vesical: Uno de los principales factores para el desarrollo de este tipo de complicación es el antecedente de cirugía prostática previa al implante. Ello se debe a que la uretra prostática y el cuello vesical se encuentran muy poco vascularizados después de la cirugía, lo que hace que toleren mal altas dosis de radiación ${ }^{47}$. Otro factor relacionado es la dosis que recibe la uretra bulbo-membranosa, de tal forma que una dosis $\leq 50 \%$ de la dosis total a dicho nivel, evitaría este tipo de complicación ${ }^{58}$. También se ha relacionado con la colocación de las semillas en posición demasiado caudal, justo anterior al ápex prostático. Técnicas de imagen de alta precisión y la colocación de una sonda vesical en el momento del implante ayuda a visualizar mejor la uretra y reducir este tipo de complicación ${ }^{47}$.

- Incontinencia urinaria: Es una de las complicaciones más limitantes. Tiene una incidencia de 0 hasta el $40 \%$ en algunas series ${ }^{12,58,60}$, aunque es raro que supere el $10 \%$ que aumenta en el caso de pacientes tratados con braquiterapia combinada con radioterapia externa y en aquellos que han sido sometidos a RTU-P, ya sea ésta realizada antes o después del implante ${ }^{60}$, si bien otros autores no han evidenciado un aumento de incidencia cuando la RTU-P es pre-braquiterapia ${ }^{61}$. Parece estar relacionada con la necrosis de la uretra y los tejidos subyacentes por la radiación ${ }^{62}$.

- Disfunción eréctil: Entre el 6 y el $53 \%$ de los pacientes sometidos a braquiterapia pueden desarrollar una disfunción eréctil después del implante $^{63}$. El mecanismo por el que se produce una disfunción eréctil post-implante es controvertido. Para DiBiase et al. ${ }^{64}$ estaría producida por la radiación que reciben los haces neurovasculares, aunque esto no ha sido confirmado por otros autores ${ }^{65}$. Merrick et al. relaciona la disfunción eréctil con la dosis recibida por el bulbo peneano, en cuyo estudio para todos los parámetros dosimétricos analizados (D25, D50, D70, D75, D90, y D95) la dosis recibida por el bulbo del pene fue estadísticamente superior en los hombres que habían desarrollado esta complicación. En el análisis multivariante la dosis recibida por el bulbo del pene y la edad del paciente en el momento del implante mostraron valor predictivo de disfunción eréctil post-implante ${ }^{66}$. Sin embargo estos hallazgos no han sido reproducidos por otros autores ${ }^{65}$. El deterioro inicial de la función eréctil debe ser atribuido al dolor y al dis- 
confort eyaculatorio, al ser el efecto de la braquiterapia gradual con el tiempo, a diferencia de la prostatectomía radical, donde el efecto es inmediato, o en la radioterapia externa, donde la función eréctil se deteriora entre los 12 y 24 meses post-radiación ${ }^{63,67}$.

Así pues, las tasas de preservación de la función eréctil están alrededor del $50-86 \%^{53,58,63}$ y la mayoría de los pacientes (83-86\%) responden al tratamiento con inhibidores de la fosfodiesterasa. Este hecho se traduce en un beneficio en cuanto a calidad de vida se refiere comparado con la prostatectomía radical, dado que en estos pacientes la respuesta a los fármacos depende de la preservación de los haces neurovasculares $^{63,66}$.

\section{b) Complicaciones digestivas crónicas:}

- Rectorragias intermitentes crónicas: Pueden aparecer en un 4-12\% de los casos y no suelen tener repercusión clínica. Son secundarias a cambios post-irradiación en la mucosa rectal cercana a la zona del implante y se controlan bien con tratamiento local sintomático ${ }^{58}$.

- Úlceras rectales y/o fistulas urinarias: Son las complicaciones más graves relacionadas con la braquiterapia pero tienen una baja incidencia $(0,3-5,4 \%)^{51,56,58,68}$. Los factores que se han visto implicados en este tipo de complicación son la dosis administrada a nivel de la mucosa rectal, la asociación con radioterapia externa y la RTU-P previo al tratamiento. Otros también asociados son la existencia de enfermedades asociadas como la enfermedad inflamatoria intestinal crónica, diabetes o HTA así como la realización de maniobras agresivas en el recto ${ }^{58,69}$. Los síntomas suelen aparecer a los 27-32 meses de media y se manifiestan como dolor ano-rectal, deposiciones con moco, diarrea, hematuria o rectorragia, emisión de orina por el recto, fecaluria e infecciones urinarias recurrentes. Debido a que es una complicación de aparición tardía, es conveniente el seguimiento de los pacientes sometidos a braquiterapia prostática por un largo período de tiempo que no debe ser inferior a los tres años ${ }^{69,70}$.

El tratamiento debe ser individualizado para cada paciente y depende del tipo de fístula desarrollada. Las posibilidades terapéuticas inclu- yen desde el manejo conservador mediante observación hasta diversos tipos de derivación intestinal y urinaria, precisando en algunos casos la realización de una cistoprostatectomía o una cistectomía ${ }^{69}$.

Un hallazgo que resulta sorprendente es el hallazgo de tumores asociados durante la reparación de la fístula. Según Yurdakul et al. el compromiso vascular inducido por la radiación produciría una isquemia con endarteritis asociada que llevaría a la formación de una fístula en la segunda o tercera década después del tratamiento. El proceso inflamatorio inducido por la braquiterapia sería un factor de riesgo para el desarrollo de nuevas neoplasias ${ }^{71,72}$.

Las principales complicaciones se recogen en la Tabla 5.

\section{CALIDAD DE VIDA TRAS BRAQUITERAPIA PROSTÁTICA}

La ABS en un reciente consenso ha identificado a la morbilidad urinaria, intestinal y sexual como aquellas áreas más frecuentemente afectadas tras el tratamiento con braquiterapia prostática. De la misma forma, recomiendan la realización de controles prospectivos de la toxicidad en estas áreas usando cuestionarios validados ${ }^{73}$. Los efectos del tratamiento dependerán de las características del paciente (edad, potencia sexual previa, etc) y del tratamiento recibido (braquiterapia sola o combinada con RT externa).

La calidad de vida de los pacientes sometidos a braquiterapia prostática ha sido evaluada comparándola con otros tratamientos. Así estudios longitudinales, prospectivos pero no randomizados, como el de Lee et al., comparan la prostatectomía radical (PR), la RT y la braquiterapia. En este estudio, bien diseñado, aunque de corto seguimiento, se evidenció un descenso de la calidad de vida (FACT-P) entre el $1^{\circ}-3^{\circ}$ meses para la braquiterapia y la $\mathrm{PR}$, aunque mayor para ésta última. A partir del $3^{\circ}$ mes y hasta el año se observó una recuperación hasta los niveles basales para ambos grupos. El grupo tratado con RT tuvo un menor descenso en la puntuación, probablemente favorecido por el corto seguimiento del estudio, al no evaluar la morbilidad tardía asociada a este tratamiento ${ }^{74}$. Fulmer et al. realizaron un estudio similar, prospectivo, longitudi- 
Tabla 5

Complicaciones de la braquiterapia de baja tasa

\begin{tabular}{|c|c|c|c|c|c|c|}
\hline Referencia & $\begin{array}{l}\text { RAO } \\
\text { n (\%) }\end{array}$ & $\begin{array}{l}\text { RTU-P } \\
\text { n (\%) }\end{array}$ & $\begin{array}{l}\text { I. urinaria } \\
\text { n (\%) }\end{array}$ & $\begin{array}{l}\text { Proctitis } \\
\text { n (\%) }\end{array}$ & $\begin{array}{l}\text { D. eréctil } \\
\text { n (\%) }\end{array}$ & $\begin{array}{c}\text { Úlceras/Fístula recal } \\
\text { n (\%) }\end{array}$ \\
\hline Prada $(2005)^{58}$ & (3) & (0) & (0) & $(2)$ & (14) & $(0,3)$ \\
\hline Aguiló (2005) ${ }^{87}$ & $6(4,35)$ & - & - & $3(2,2)$ & - & - \\
\hline Wallner (1996) 56 & $13(14)$ & $8(8,7)$ & $6(6,5)$ & $5(5,4)$ & $13(14)$ & $5(5,4)$ \\
\hline Terk $(1998)^{12}$ & $14(5,5)$ & $6(2,4)$ & - & - & - & - \\
\hline Benoit $(2000)^{68}$ & $205(14,5)$ & $122(8,7)$ & $95(6,7)$ & $94(6,7)$ & $90(6,3)$ & $21(1,5)$ \\
\hline Gelblum $(1999)^{50}$ & - & $28(4,7)$ & $4(0,7)$ & - & - & - \\
\hline Sarosdy $(2004)^{51}$ & $24(24)$ & $5(5,2)$ & - & $15(15,5)$ & - & - \\
\hline Merrick $(2000)^{8}$ & $10(6)$ & $2(1,2)$ & $0(0)$ & - & - & - \\
\hline Guinot $(2005)^{\wedge 88}$ & $9(7)$ & $2(1,5)$ & $1(0,7)$ & $0(0)$ & $(30)^{*}$ & $1(0,7)$ \\
\hline Tormo $(2006)^{\wedge}$ & $6(6,25)$ & $2(2,08)$ & $1(1,04)$ & $0(0)$ & $24(25)^{*}$ & $0(0)$ \\
\hline
\end{tabular}

* Con función eréctil previa preservada.

$\wedge$ Datos no publicados

nal, no randomizado, pero con evaluación de síntomas basales (urinarios y sexuales) previo al tratamiento. Los pacientes sometidos a PR tuvieron las mejores puntuaciones basales (previas al tratamiento) en función sexual y urinaria seguido del grupo de pacientes sometidos a braquiterapia y a braquiterapia combinada con RT. La peor función urinaria correspondió a los pacientes con PR (incontinencia urinaria), pero con repercusión similar en calidad de vida que los sometidos a RT y braquiterapia probablemente debido a los sintomas irritativos presentes en éstos últimos. El grupo de PR tuvo la peor función sexual, comparable al grupo de braquiterapia combinada con RT a los 18 meses de seguimiento. El grupo sometido a PR fue el de menor recuperación porcentual con respecto a los valores basales en función sexual y urinaria a lo largo de todo el seguimiento ${ }^{75}$. Otros estudios, de diseño transversal y sin cuestionarios basales, como el de Brandeis et al., no evidenciaron diferencias en calidad de vida (UCLA, PCI) entre distintos grupos de tratamiento $(\mathrm{PR}$, braquiterapia, braquiterapia más radioterapia y controles), con una mediana de seguimiento de 7,5 meses. La función urinaria fue mejor en controles, en pacientes sometidos a braquiterapia y peor en pacientes tratados con PR y braquiterapia combinada con radioterapia. La función intestinal fue peor en el grupo de braquiterapia combinada con radioterapia y la función sexual fue preservada en el grupo de braquitera- pia y peor pero similar en puntuación entre el grupo de PR y braquiterapia más radioterapia ${ }^{76}$. En un estudio similar, de mayor seguimiento (2237 meses), la braquiterapia mostró mayor sintomatología urinaria (mayor puntuación del IPSS) que la $\mathrm{PR}$, pero sin embargo mostró mejor funcionalidad sexual y urinaria. La braquiterapia combinada con radioterapia mostró peor función intestinal, pero mejor función sexual y urinaria que la $\mathrm{PR}^{77}$.

Sin embargo, no existe actualmente suficiente evidencia científica que nos permita contestar adecuadamente a la pregunta de cual es el tratamiento que menor toxicidad produce para el cáncer de próstata organoconfinado. Son necesarios más estudios longitudinales y randomizados, que permitan definir aquel tratamiento que repercuta menos en la calidad de vida. Sin embargo a la hora de seleccionar una opción de tratamiento por parte del paciente, tendrá un poderoso peso específico los efectos secundarios esperables de dicha opción. Los datos de los que disponemos actualmente, nos indican que la braquiterapia es probablemente la opción que menos repercute en la función sexual y que menor incontinencia produce, pero a expensas de un incremento de los LUTS en el primer año postratamiento. Probablemente los resultados de SPIRIT (surgical prostatectomy vs. Intersticial radiotherpy Intervention Trial) aclare o conteste todas estas cuestiones $^{78}$. 


\section{REFERENCIAS}

1. Blasko JC, Mate T, Sylvester JE, Grimm PD, Cavanagh W. Brachytherapy for carcinoma of the prostate: techniques, patient selection, and clinical outcomes. Semin Radiat Oncol 2002;12(1):81-94.

2. Pasteau O. Traitment du cancer de la prostate par le Radium. Rev Malad Nutr 1911:363-367.

3. Whitmore WF, Jr., Hilaris B, Grabstald H. Retropubic implantation to iodine 125 in the treatment of prostatic cancer. J Urol 1972;108(6):918-920.

4. Carlton CE, Jr., Dawoud F, Hudgins P, Scott R, Jr. Irradiation treatment of carcinoma of the prostate: a preliminary report based on 8 years of experience. J Urol 1972;108 (6):924-927.

5. Holm HH, Juul N, Pedersen JF, Hansen H, Stroyer I. Transperineal 125iodine seed implantation in prostatic cancer guided by transrectal ultrasonography. J Urol 1983;130(2):283-286.

6. Stone NN, Stock RG. Brachytherapy for prostate cancer: real-time three-dimensional interactive seed implantation. Tech Urol 1995;1(2):72-80.

7. Nag S, Beyer D, Friedland J, Grimm P, Nath R. American Brachytherapy Society (ABS) recommendations for transperineal permanent brachytherapy of prostate cancer. Int J Radiat Oncol Biol Phys 1999;44(4):789-799.

8. Merrick G, Butler W, Lief J, Dorsey A. Temporal resolution of urinary morbidity following prostate brachytherapy. Int J Radiat Oncol Biol Phys 2000;47:121-128

9. tone NN, Stock RG. Prostate brachytherapy in patients with prostate volumes $>/=50 \mathrm{~cm}(3)$ : dosimetic analysis of implant quality. Int J Radiat Oncol Biol Phys 2000;46(5): 1199-1204.

10. Merrick GS, Butler WM, Dorsey AT, Lief JH. Effect of prostate size and isotope selection on dosimetric quality following permanent seed implantation. Tech Urol 2001;7(3): 233-240.

11. Merrick GS, Butler WM, Wallner KE, Galbreath RW, Adamovich E. Permanent interstitial brachytherapy in younger patients with clinically organ-confined prostate cancer. Urology 2004;64(4):754-759.

12. Terk M, Stock R, Stone NN. Identification of patients at increased risk for prolonged urinary retention following radioactive seed implantation of the prostate. J Urol 1998; 160:1379-1382.

13. Merrick GS, Butler WM, Wallner KE, Lief JH, Galbreath RW. Prophylactic versus therapeutic alpha-blockers after permanent prostate brachytherapy. Urology 2002;60(4): 650-655.

14. Landis D, Wallner K, Locke J, Ellis W, Russell K, Cavanagh $\mathrm{W}$, et al. Late urinary function after prostate brachytherapy. Brachytherapy 2002;1(1):21-26.

15. Hughes S, Wallner K, Merrick G, Miller S, True LD. Preexisting histologic evidence of prostatitis is unrelated to postimplant urinary morbidity. Int $J$ Cancer 2001;96 Suppl:79-82.

16. Wallner K, Lee H, Wasserman S, Dattoli M. Low risk of urinary incontinence following prostate brachytherapy in patients with a prior transurethral prostate resection. Int J Radiat Oncol Biol Phys 1997;37(3):565-569.

17. Davis BJ, Pisansky TM, Wilson TM, Rothenberg HJ, Pacelli A, Hillman DW, et al. The radial distance of extraprostatic extension of prostate carcinoma: implications for prostate brachytherapy. Cancer 1999;85(12):2630-2637.
18. Blasko JC, Grimm PD, Sylvester JE, Badiozamani KR, Hoak D, Cavanagh W. Palladium-103 brachytherapy for prostate carcinoma. Int J Radiat Oncol Biol Phys 2000;46 (4):839-850.

19. Dattoli M, Wallner K, True L, Sorace R, Koval J, Cash J, et al. Prognostic role of serum prostatic acid phosphatase for 103Pd-based radiation for prostatic carcinoma. Int $\mathrm{J}$ Radiat Oncol Biol Phys 1999;45(4):853-856.

20. Merrick GS, Butler WM, Lief JH, Galbreath RW, Adamovich $\mathrm{E}$. Biochemical outcome for hormone-naive patients with high-risk prostate cancer managed with permanent interstitial brachytherapy and supplemental externalbeam radiation. Cancer J 2002;8(4):322-327.

21. Valicenti R, Lu J, Pilepich M, Asbell S, Grignon D. Survival advantage from higher-dose radiation therapy for clinically localized prostate cancer treated on the Radiation Therapy Oncology Group trials. J Clin Oncol 2000;18(14):2740-2746.

22. Do TM, Parker RG, Smith RB, Kagan AR. High-grade carcinoma of the prostate: a comparison of current local therapies. Urology 2001;57(6):1121-1126; discussion 1126-1127.

23. Merrick GS, Wallner KE, Butler WM. Permanent interstitial brachytherapy for the management of carcinoma of the prostate gland. J Urol 2003;169(5):1643-1652.

24. Merrick GS, Butler WM, Wallner KE, Galbreath RW, Allen $\mathrm{Z}$, Lief $\mathrm{JH}$, et al. Influence of body mass index on biochemical outcome after permanent prostate brachytherapy. Urology 2005;65(1):95-100.

25. Grann A, Wallner K. Prostate brachytherapy in patients with inflammatory bowel disease. Int J Radiat Oncol Biol Phys 1998;40(1):135-138.

26. Porter AT, Blasko JC, Grimm PD, Reddy SM, Ragde H. Brachytherapy for prostate cancer. CA Cancer J Clin 1995; 45(3): 165-178.

27. Stock RG, Stone NN, DeWyngaert JK, Lavagnini P, Unger PD. Prostate specific antigen findings and biopsy results following interactive ultrasound guided transperineal brachytherapy for early stage prostate carcinoma. Cancer 1996;77(11):2386-2392.

28. Merrick GS, Butler WM, Dorsey AT, Lief JH. The dependence of prostate postimplant dosimetric quality on CT volume determination. Int J Radiat Oncol Biol Phys 1999; 44(5): 1111-1117.

29. Badiozamani KR, Wallner K, Cavanagh W, Blasko J. Comparability of CT-based and TRUS-based prostate volumes. Int J Radiat Oncol Biol Phys 1999;43(2):375-378

30. Stone NN, Stock RG. Prostate brachytherapy: treatment strategies. J Urol 1999;162(2):421-426.

31. Merrick GS, Butler WM, Lief JH, Dorsey AT. Is brachytherapy comparable with radical prostatectomy and externalbeam radiation for clinically localized prostate cancer? Tech Urol 2001;7(1):12-19.

32. Grimm PD, Blasko JC, Sylvester JE, Meier RM, Cavanagh W. 10-year biochemical (prostate-specific antigen) control of prostate cancer with (125)I brachytherapy. Int J Radiat Oncol Biol Phys 2001;51(1):31-40.

33. Zelefsky MJ, Hollister T, Raben A, Matthews S, Wallner KE. Five-year biochemical outcome and toxicity with transperineal CT-planned permanent I-125 prostate implantation for patients with localized prostate cancer. Int J Radiat Oncol Biol Phys 2000;47(5):1261-1266.

34. Potters L, Morgenstern C, Calugaru E, Fearn P, Jassal A, Presser J, et al. 12-year outcomes following permanent prostate brachytherapy in patients with clinically localized prostate cancer. J Urol 2005;173(5):1562-1566. 
35. Merrick GS, Butler WM, Galbreath RW, Lief JH. Five-year biochemical outcome following permanent interstitial brachytherapy for clinical T1-T3 prostate cancer. Int $\mathrm{J}$ Radiat Oncol Biol Phys 2001;51(1):41-48.

36. Critz FA, Williams WH, Levinson AK, Benton JB, Holladay CT, Schnell FJ, Jr. Simultaneous irradiation for prostate cancer: intermediate results with modern techniques. J Urol 2000;164(3 Pt 1):738-41; discussion 741-743.

37. Blasko JC, Wallner K, Grimm PD, Ragde H. Prostate specific antigen based disease control following ultrasound guided 125iodine implantation for stage $\mathrm{T} 1 / \mathrm{T} 2$ prostatic carcinoma. J Urol 1995; 154(3): 1096-1099.

38. D'Amico AV, Whittington R, Malkowicz SB, Schultz D, Blank K, Broderick GA, et al. Biochemical outcome after radical prostatectomy, external beam radiation therapy, or interstitial radiation therapy for clinically localized prostate cancer. Jama 1998;280(11):969-974.

39. Kwok Y, DiBiase SJ, Amin PP, Naslund M, Sklar G, Jacobs SC. Risk group stratification in patients undergoing permanent (125)I prostate brachytherapy as monotherapy. Int J Radiat Oncol Biol Phys 2002;53(3):588-594.

40. Battermann JJ, Boon TA, Moerland MA. Results of permanent prostate brachytherapy, 13 years of experience at a single institution. Radiother Oncol 2004;71(1):23-8.

41. Wallner K, Blasko J, Dattoli M. Prostate Brachytherapy made complicated. 2nd ed. Seattle; 2001.

42. Brachman DG, Thomas T, Hilbe J, Beyer DC. Failure-free survival following brachytherapy alone or external beam irradiation alone for T1-2 prostate tumors in 2222 patients: results from a single practice. Int $\mathrm{J}$ Radiat Oncol Biol Phys 2000;48(1):111-117.

43. Stone NN, Stock RG, Unger P. Intermediate term biochemical-free progression and local control following 125iodine brachytherapy for prostate cancer. J Urol 2005;173(3): 803-837.

44. Bolla M, Gonzalez D, Warde P, Dubois JB, Mirimanoff RO, Storme G, et al. Improved survival in patients with locally advanced prostate cancer treated with radiotherapy and goserelin. N Engl J Med 1997;337(5):295-300.

45. Lee LN, Stock RG, Stone NN. Role of hormonal therapy in the management of intermediate- to high-risk prostate cancer treated with permanent radioactive seed implantation. Int J Radiat Oncol Biol Phys 2002;52(2):444-452.

46. Merrick GS, Butler WM, Wallner KE, Galbreath RW, Anderson RL. Prostate-specific antigen spikes after permanent prostate brachytherapy. Int J Radiat Oncol Biol Phys 2002;54(2):450-456.

47. Stone N, Stock R. Complications following permanent prostate brachytherapy. Eur Urol 2002;41:427-433.

48. Mallick S, Azzouzi R, Cormier L, Peiffert D, Mangin P. Urinary morbidity after 125 I brachytherapy of the prostate. BJU 2003;92:555-558.

49. Desai J, Stock RG, Stone NN, Iannuzzi C, DeWyngaert JK. Acute urinary morbidity following I-125 interstitial implantation of the prostate gland. Radiat Oncol Investig 1998; 6(3): 135-141.

50. Gelblum D, Potters L, Ashley R, Waldbaum R, Wang X, Leibel S. Urinary morbidity following ultrasound-guided transperineal prostate seed implantation. Int $\mathrm{J}$ Radiat Oncol Biol Phys 1999;45:59-67.

51. Sarosdy M. Urinary and rectal complications of contemporary permanent transperineal brachytherapy for prostate carcinoma with or. Cancer 2004;101:754-760.
52. Crook J, McLean M, Catton C, Yeung I, Tsihlias J, Pintilie M. Factors influencing risk of acute urinary retention after TRUS-guided permanent prostate seed implantation. Int $\mathrm{J}$ Radiat Oncol Biol Phys 2002;52(2):453-460.

53. Locke J, Ellis W, Wallner K, Cavanagh W, Blasko J. Risk factors for acute urinary retention requiring temporary intermittent catheterization after prostate brachytherapy: a prospective study. Int $J$ Radiat Oncol Biol Phys 2002; 52(3):712-719.

54. Kollmeier MA, Stock RG, Cesaretti J, Stone NN. Urinary morbidity and incontinence following transurethral resection of the prostate after brachytherapy. J Urol 2005; 173(3):808-812.

55. Hirshberg ED, Klotz LH. Post transurethral resection of prostate incontinence in previously radiated prostate cancer patients. Can J Urol 1998;5(2):560-563.

56. Wallner K, Roy J, Harrison L. Tumor control and morbidity following transperineal iodine 125 implantation for stage T1/T2 prostatic carcinoma. J Clin Oncol 1996;14:449-453.

57. Pérez Romasanta L. Grados de toxicidad de la RTOG/ EORTC.

58. Prada P, Hevia M, Juan G, Abascal J, De la Rua A, Abascal $\mathrm{R}$ et al. Braquiterapia de baja tasa (I125) en el cáncer de prostata localizado. Preliminary results after 5 years. Arch Esp Urol 2005;58:213-226.

59. Wallner K, Roy J, Harrison L. Dosimetry guidelines to minimize urethral and rectal morbidity following transperineal I-125 prostate brachytherapy. Int J Radiat Oncol Biol Phys 1995;32(2):465-471.

60. Talcott J, Clark J, Stark P, Mitchell S. Long-term treatment related complications of brachytherapy for early prostate cancer: a survey of patients previously treated. $\mathrm{J}$ Urol 2001;166:494-499.

61. Stone N, Ratnow E, Stock R. Prior transurethral resection does not increase morbidity following real-time ultrasound-guided prostate seed implantation. Tech Urol 2000:123-127.

62. Blasko JC, Radge H, Grimm PD. Transperineal ultrasound-guided implantation of the prostate: Morbidity and complications. Scand J Urol Nephrol 1991;137:113-118.

63. Mabjeesh N, Chen J, Beri A, Stenger A, Matzkin H. Sexual function after permanent 125I-brachytherapy for prostate cancer. Int J Impot Res 2005; 17:96-101.

64. DiBiase S, Wallner K, Tralins K, Sutlief S. Brachytherapy radiation doses to the neurovascular bundles. Int $J$ Radiat Oncol Biol Phys 2000;46:1301-1307.

65. Kiteley R, Lee W, deGuzman A, Mirzaei M, Mc Cullough D. Radiation dose to the neurovascular bundles or penile bulb does not predict erectile dysfunction after prostate brachytherapy. Brachytherapy 2002;1:90-94.

66. Merrick G, Wallner K, Butler W, Galbreath R, Lief J, Benson M. A comparision of radiation doe to the bulb of the penis in men with and without prostate brachytherapy-induced erectile dysfunction. Int $\mathrm{J}$ Radiat Oncol Biol Phys 2001;50:597-604.

67. Robinson J, Moritz S, Fung T. Meta-analysis of rates of erectile function after treatment of localized prostate carcinoma. Int J Radiat Oncol Biol Phys 2002;54:1063-1068.

68. Benoit R, Naslund M, Cohen J. Complications after prostate brachyterapy in the medicare population. Urol 2000; 55:91-96.

69. Chrouser K, Leibovich B, Sweat S, Larson D, BJ. D, Tran $\mathrm{N}$. Urinary fistulas following external radiation or permanent brachytherapy for the treatment of prostate cancer. J Urol 2005; 173:1953-1957. 
70. Shah S, Cima R, Benoit E, Breen E, Bleday R. Rectal complications after prostate brachytherapy. Dis Colon rectum 2004;47:1487-1492.

71. Moreira S, Seigne J, Ondorica R, Marcel J, Pow-sang J, Lockhart J. Devasting complications after brachytherapy in the treatment of prostate adenocarcinoma. BJU 2004; 93:31-35.

72. Yurdakul G, de Reijke T, Blank L, Rauws E. Rectal squamous cell carcinoma 11 years after brachytherapy for carcinoma of the prostate. J Urol 2003;169:280-285.

73. Nag S, Ellis RJ, Merrick GS, Bahnson R, Wallner K, Stock R. American Brachytherapy Society recommendations for reporting morbidity after prostate brachytherapy. Int $\mathrm{J}$ Radiat Oncol Biol Phys 2002;54(2):462-470.

74. Lee WR, Hall MC, McQuellon RP, Case LD, McCullough DL. A prospective quality-of-life study in men with clinically localized prostate carcinoma treated with radical prostatectomy, external beam radiotherapy, or interstitial brachytherapy. Int J Radiat Oncol Biol Phys 2001;51(3): 614-623.

75. Fulmer BR, Bissonette EA, Petroni GR, Theodorescu D. Prospective assessment of voiding and sexual function after treatment for localized prostate carcinoma: comparison of radical prostatectomy to hormonobrachytherapy with and without external beam radiotherapy. Cancer 2001;91(11):2046-2055.

76. Brandeis JM, Litwin MS, Burnison CM, Reiter RE. Quality of life outcomes after brachytherapy for early stage prostate cancer. J Urol 2000;163(3):851-857.

77. Davis JW, Kuban DA, Lynch DF, Schellhammer PF. Quality of life after treatment for localized prostate cancer: differences based on treatment modality. J Urol 2001;166 (3):947-952.

78. Henderson A, Laing RW, Langley SE. Quality of life following treatment for early prostate cancer: does low dose rate (LDR) brachytherapy offer a better outcome? A review. Eur Urol 2004;45(2):134-141.

79. Beyer DC, Priestley JB, Jr. Biochemical disease-free survival following 125I prostate implantation. Int $\mathrm{J}$ Radiat Oncol Biol Phys 1997;37(3):559-563.

80. Stokes SH, Real JD, Adams PW, Clements JC, Wuertzer S, Kan W. Transperineal ultrasound-guided radioactive seed implantation for organ-confined carcinoma of the prostate. Int J Radiat Oncol Biol Phys 1997;37(2):337-341.

81. Grado GL, Larson TR, Balch CS, Grado MM, Collins JM, Kriegshauser JS, et al. Actuarial disease-free survival after prostate cancer brachytherapy using interactive techniques with biplane ultrasound and fluoroscopic guidance. Int J Radiat Oncol Biol Phys 1998;42(2):289-298.
82. Sharkey J, Chovnick SD, Behar RJ, Perez R, Otheguy J, Solc Z, et al. Outpatient ultrasound-guided palladium 103 brachytherapy for localized adenocarcinoma of the prostate: a preliminary report of 434 patients. Urology 1998;51 (5):796-803

83. Storey MR, Landgren RC, Cottone JL, Stallings JW, Logan CW, Fraiser LP, et al. Transperineal 125iodine implantation for treatment of clinically localized prostate cancer: 5year tumor control and morbidity. Int $\mathrm{J}$ Radiat Oncol Biol Phys 1999;43(3):565-570.

84. Ragde H, Korb LJ, Elgamal AA, Grado GL, Nadir BS. Modern prostate brachytherapy. Prostate specific antigen results in 219 patients with up to 12 years of observed follow-up. Cancer 2000;89(1):135-141.

85. Ragde H, Grado GL, Nadir BS. Brachytherapy for clinically localized prostate cancer: thirteen-year disease-free survival of 769 consecutive prostate cancer patients treated with permanent implants alone. Arch Esp Urol 2001;54(7): 739-747.

86. Merrick GS, Butler WM, Wallner KE, Galbreath RW, Adamovich E. Monotherapeutic brachytherapy for clinically organ-confined prostate cancer. W V Med J 2005;101 (4): 168-171.

87. Aguilo Lucia F, Suarez Novo JF, Correa Generoso R, Boladeras Inglada AM, Polo Rubio A, Pera Fabregas J, et al. Estudio retrospectivo de 130 pacientes con cáncer prostático organoconfinado tratados con braquiterapia prostática. Actas Urol Esp 2005;29(1):47-54.

88. Guinot JL, Estornell R, Escolar PP. What have we learned in the first two years about I-125 prostate brachytherapy? In: ESTROCongress, editor. Budapest: Radiother Oncol 75 supl (2005) S-36;2005.

89. Hu K, Wallner K. Urinary incontinence in patients who have a TURP/TUIP following prostate brachytherapy. Int $\mathrm{J}$ Radiat Oncol Biol Phys 1998;40(4):783-186.

90. Koutrouvelis PG, Lailas N, Katz S, Sehn J, Gil-Montero G, Khawand N. Prostate cancer with large glands treated with 3-dimensional computerized tomography guided pararectal brachytherapy: up to 8 years of followup. J Urol 2003; 169(4):1331-1336.

Correspondencia: Dr. A. Budía Alba

Servicio de Urología. Hospital Universitario La Fe

Avda. Campanar, 21. 46009 Valencia

Tel.: 963862700 Ext 40686

E-mail autor: budia@pulso.com

Información artículo: Revisión - Cáncer de próstata

Trabajo recibido: febrero 2007

Trabajo aceptado: abril 2007 